\title{
A comparison of outcomes with coronary artery calcium scanning in Unselected Populations - The Multi-Ethnic Study of Atherosclerosis (MESA) and Heinz Nixdorf Recall Study (HNR)
}

\author{
MJ Budoff, MD ${ }^{1}$, Stefan Möhlenkamp, MD², Robyn McClelland, PhD $^{3}$, Joseph A. Delaney, \\ $\mathrm{PhD}^{4}$, Marcus Bauer, MD ${ }^{2}$, Heinz Karl Jöckel, PhD ${ }^{5}$, Hagen Kälsch, MD ${ }^{5}$, Richard Kronmal, \\ $\mathrm{PhD}^{3}$, Khurram Nasir, MD, MPH ${ }^{6}$, Nils Lehmann, $\mathrm{PhD}^{5}$, Susanne Moebus, $\mathrm{PhD}, \mathrm{MPH}^{5}$, Ken \\ Mukamal, MD MPH ${ }^{7}$, and Raimund Erbel, $\mathrm{MD}^{2}$ on behalf of the Multi-Ethnic Study of \\ Atherosclerosis and the Investigator Group of the Heinz Nixdorf Recall Study \\ ${ }^{1}$ Los Angeles Biomedical Research Center, Torrance, CA 90502, USA \\ ${ }^{2}$ Department of Cardiology, University of Duisburg-Essen, Hufelandstraßfe 55, D-45122 Essen, \\ Germany \\ ${ }^{3}$ Department of Biostatistics, University of Washington, Seattle, WA 98115, USA \\ ${ }^{4}$ College of Pharmacy, University of Florida, FL, USA \\ ${ }^{5}$ Institut of Medical Informatics, Biometry, and Epidemiology, University of Duisburg-Essen, \\ Essen, Germany \\ ${ }^{6}$ Tufts Medical Center, Departments of Radiology and CardioVascular Center, Boston MA, USA \\ ${ }^{7}$ Division of General Medicine and Primary Care, Beth Israel Deaconess Medical Center, Boston, \\ MA 02215, USA
}

\section{Abstract}

Background-The Multi-Ethnic Study of Atherosclerosis (MESA) and the Heinz Nixdorf Recall Study (HNR)) differed in regards to informing physicians and patients of the results of their subclinical atherosclerosis.

Objective-This study investigates whether the association of coronary artery calcium (CAC) with incident non-fatal and fatal cardiovascular (CVD) events is different among these two large, population-based observational studies.

\begin{abstract}
Methods-All Caucasian subjects aged 45-75 years, free of baseline cardiovascular disease were included ( $\mathrm{n}=2232$ in MESA, $\mathrm{n}=3119$ HNR participants). We studied the association between CAC and event rates at 5 years, including hard cardiac events (MI, cardiac death, resuscitated cardiac arrest), and separately added revascularizations, and strokes (fatal and non-fatal) to determine adjusted hazard ratios (HR).
\end{abstract}

Results-Both cohorts demonstrated very low CHD (including revascularization) rates with zero calcium (1.13 and $1.16 \%$ over 5 years in MESA and HNR respectively) and increasing

(C) 2013 Society for Cardiovascular Computed Tomography. Published by Elsevier Inc. All rights reserved.

Correspondence author: Matthew Budoff, MD, Los Angeles Biomedical Research Institute, 1124 W Carson St, Torrance, CA 90502, mbudoff@labiomed.org.

Publisher's Disclaimer: This is a PDF file of an unedited manuscript that has been accepted for publication. As a service to our customers we are providing this early version of the manuscript. The manuscript will undergo copyediting, typesetting, and review of the resulting proof before it is published in its final citable form. Please note that during the production process errors may be discovered which could affect the content, and all legal disclaimers that apply to the journal pertain. 
significantly in both groups with CAC 100-399 (6.71 and 4.52\% in MESA and HNR) and CAC $>400$ (12.5 and 13.54\% in MESA and HNR respectively) and demonstrating strong independent predictive values for scores of 100-399 and >400, despite multivariable adjustment for risk factors. Risk factor adjusted five year revascularization rates were nearly identical for HNR and MESA, and generally low for both studies (1.4\% [45/3119] for HNR and 1.9\% [43/2232] for MESA) over 5 years.

Conclusions-Across two culturally diverse populations, CAC $>400$ is a strong predictor of events. High CAC did not determininistically result in revascularization and knowledge of CAC did not increase revascularizations.

\section{Keywords}

coronary artery calcification; subclinical atherosclerosis; Multi-Ethnic Study of Atherosclerosis (MESA); Heinz Nixdorf Recall Study (HNR)

\section{Introduction}

International guidelines currently incorporate coronary artery calcium (CAC) testing in both diagnostic algorithms for chest pain and risk stratification for asymptomatic patients ${ }^{1,2}$ based upon studies demonstrating its relationship to cardiovascular outcomes. ${ }^{2,3}$ Multicenter studies and registries indicate that the risk for myocardial infarction and cardiovascular death (hard events) during follow-up is increased with increasing CAC scores. ${ }^{4-8}$

Two prospective studies are currently underway; the MESA (uutti-Ethnic $\underline{\text { Study of }}$ $\underline{\text { Atherosclerosis) }}{ }^{5}$ and the Heinz Nixdorf Recall Study (HNR; Risk factors, $\underline{\text { Evaluation of }}$ Coronary Calcium and $\underline{\text { Lifestyle Factors). }}{ }^{6}$ Both of these population-based studies have measured $\overline{\mathrm{CAC}}$ to quantify subclinical atherosclerosis at baseline entry. ${ }^{7,8}$ This paper combines and compares results from subsets of the HNR study, conducted in the Ruhr area cities of Bochum, Essen, and Mülheim, in Germany, and MESA, conducted in six urban areas of the United States, to evaluate CAC and CV outcomes, and its association with risk factors in two different unselected populations without clinical cardiovascular disease. A cross sectional comparison of the baseline characteristics and calcium scores of the participants (free of clinical cardiovascular disease and of European descent) has been previously been published. ${ }^{9-12}$

One of the biggest methodological differences in the conduct of the studies relates to informing participants and study doctors of the results of the baseline examinations, including the measures of subclinical disease. In MESA, an initial report summarizes results available at the completion of the clinic visit and results of computed tomography, magnetic resonance imaging, and ultrasonography are reported by mail. In the HNR study, all findings except the Electron Beam Tomography results and experimental findings, such as some novel risk factors or genetic polymorphisms, are reported to the participants and, when they agree, their primary physicians. It was our aim to investigate the outcomes over 5 years of follow-up regarding CAC for an asymptomatic Caucasian population in the United States and Germany, and evaluate the impact of differences in risk factors possibly affecting CACdependent cardiovascular outcomes.

\section{Methods}

\section{Study populations}

The MESA study recruited 6814 participants between the years 2000 and 2002 across six centers in the United States with participants recruited using locally available resources, 
including lists of residents, dwellings, telephone exchanges, division of motor vehicle lists, consumer lists, voter registration lists, and census data. Each site recruited an approximately equal number of men and women, according to pre-specified age and race/ethnicity proportions. Participants were between 45 and 84 years of age and self-identified themselves as one of Caucasian, African-American, Hispanic, or Chinese descent. ${ }^{9}$ For this comparison of the two study cohorts, all Caucasian subjects aged 45-75 years, free of baseline cardiovascular disease were included ( $\mathrm{n}=2232$ in MESA). In MESA, CAC scores were obtained via Electron Beam Tomography or multi-detector computed tomography. Covariate information was missing in 14 participants and follow up data not available in 9 participants, for a total sample size of 2209 (Figure 1).

The HNR recruited a total of 4814 Caucasian participants between 45 and 75 years of age from three neighbouring cities in Germany between the years 2000 and 2003 in a single center with a response rate of 55.8\%. Participants were a random sample derived from mandatory citizen registries and provided to the study center. ${ }^{10}$ All participants underwent Electron Beam Computed Tomography (EBCT) scanning to derive CAC scores. For this comparison, only members of HNR who were free of clinical cardiovascular disease at baseline with both CAC scans and follow up information available were included. These exclusions resulted in a total of 3119 eligible participants (Figure 2). The study was certified according to DIN EN ISO 9001:2000, and re-certified in 2006.

Therefore, between the two cohorts, a total of 5335 asymptomatic male and female participants were included. All participants had CAC scanning of the heart and longitudinal follow up for at least 5 years. For both studies, ethics approval was provided by the local institutional review boards.

\section{Clinical data}

The traditional cardiovascular risk factors, defined as those that are part of the Framingham risk scoring algorithm ${ }^{10}$, were measured in both studies. In addition, body mass index (BMI; $\mathrm{kg} / \mathrm{m}^{2}$ ) was computed on the basis of direct measurements of height and weight. All medication utilization information was based on participants' self-report or medication inventory, including anti-hypertensive and lipid lowering medications (including HMG CoA reductase inhibitors, fibrates, bile acid sequestrants, and nicotinic acid derivatives). Standard enzymatic methods were used to measure total cholesterol, high-density lipoprotein (HDL)cholesterol (HDL-C), and triglycerides. ${ }^{9}{ }^{9} 10$ Low-density lipoprotein (LDL)-cholesterol (LDL-C) was calculated with the Friedewald equation in MESA ${ }^{13}$ and measured directly in HNR. ${ }^{14}$ Blood samples were obtained after a 12 hours (h) fasting in MESA. In HNR, participants were fasting $9.7+4.9 \mathrm{~h}$ (median $12 \mathrm{~h}$ ) before blood sampling, with $34.4 \%$ having fasted for $6 \mathrm{~h}$. In both studies, blood pressure was measured three times, two minutes apart, using an oscillographic method with two different systems (Dynamap ${ }^{\circledR}$, J\&J, USA and HEM-705CP, Omron, Hoofddrop, NL). The mean values of the second and third of three measurements were used to estimate clinical blood pressure. Hypertension was defined in both studies as blood pressure $>140 / 90 \mathrm{mmHg}$ or use of antihypertensive medication. Participants were considered diabetic if they were taking anti-diabetic medication or had a measured fasting glucose of $>126 \mathrm{mg} / \mathrm{dL}$. Smoking history was categorized to (i) currently smoking, (ii) former, defined as not smoking within the past 30 days in MESA and as stopped smoking (a) within the past year or (b) more than 1 year ago in the HNR, and (iii) never.

\section{Measurement of coronary artery calcium}

Electron beam and multi-slice computed tomography-Non-enhanced EBCT scans were performed with a C-100 or C-150 scanner (GE Imatron, South San Francisco, 
USA) in 3 MESA and the HNR centers. In addition 3 other MESA centers used a first generation multislice computed tomogram. The EBCT scanners were operated in the single slice mode with an image acquisition time of 100 milliseconds and a section thickness of 3 $\mathrm{mm}$. Prospective electrocardiographic triggering was done at $80 \%$ of the R-R interval for EBCT. Each participant was scanned twice in MESA and once in HNR. ${ }^{12}$ In the MESA study, scans were read centrally at the Los Angeles Biomedical Research Institute at HarborUCLA Medical Center. In the HNR study, the two radiology departments scanned and analyzed the CAC score in a blinded fashion and independently. In both studies, CAC was defined as a hyper-attenuating foci of at least 4 contiguous pixels with a CT density $\geq 130$ Hounsfield Unit for determination of a calcium score. ${ }^{11}$ The CAC score is the product of the area of each focus of detectable CAC and a factor rated 1 through 4 dictated by the maximum CT density within that focus. The total CAC score was computed by summing-up the $\mathrm{CAC}$ scores of all foci in the epicardial coronary system without phantom adjustment. Agreement with regard to presence of CAC was high in the MESA study ( $\kappa$ - value 0.90 0.93 ) and interclass correlation coefficient for CAC scoring of 0.99. In the HNR study, interscan variability was $5-8 \%$, and for inter-institutional readings of the 2 EBCT centers a $x$ value of 0.94 in 250 scans was found. ${ }^{12}$

CVD Follow-Up-Five year follow up data for incident CVD events was used in both cohorts. In MESA, at intervals of 9-12 months, a telephone interviewer contacted each participant to inquire about interim hospital admissions, cardiovascular outpatient diagnoses, and deaths. Copies of all death certificates and medical records for hospitalizations and outpatient cardiovascular diagnoses were obtained and reviewed by a central adjudication team. We also conducted next-of-kin interviews for out of hospital cardiovascular deaths. We obtained records on an estimated $98 \%$ of reported hospitalized cardiovascular events and some information on $95 \%$ of reported outpatient diagnostic encounters.

Two physicians independently reviewed and classified CVD events and assigned incidence dates. If, after review and adjudication, disagreements persisted, a full mortality and morbidity review committee made the final classification. Reviewers classified myocardial infarction as definite, probable or absent, based primarily on combinations of symptoms, ECG, and cardiac biomarker levels. They classified coronary heart disease (CHD) or CVD death as present or absent based on hospital records and interviews with families. Definite fatal CHD required an MI within 28 days of death, chest pain within the 72 hours before death, or a history of CHD and the absence of a known non-atherosclerotic or non-cardiac cause of death. Neurologists reviewed and classified stroke as present if there was a focal neurologic deficit lasting 24 hours or until death, with a clinically relevant lesion on brain imaging, and no nonvascular cause.

For this report, hard CVD was defined as CHD (definite and probable myocardial infarction, definite coronary heart disease death, resuscitated cardiac arrest), stroke (fatal or nonfatal), or other atherosclerotic CVD death. Follow-up went from the baseline examination until the first CVD event, loss to follow-up, death, or else for a total follow up of 5 years in both studies.

\section{Event Ascerntainment in HNR}

\section{Study end points and verification of study end points}

Primary end points for this study were based on unequivocally documented incident coronary events that met predefined study criteria. This study classified a myocardial infarction event based on symptoms, signs of electrocardiography, and enzymes (levels of creatine kinase), as well as troponin T or I, and necropsy as nonfatal acute myocardial infarction and coronary death ${ }^{13}$. For all primary study end points, hospital and nursing 
home records, including electrocardiograms, laboratory values, and pathology reports, were collected. For deceased subjects, death certificates were collected and interviews with general practitioners, relatives, and eyewitnesses were undertaken if possible. Medical records were obtained in $100 \%$ of all reported end points. An external end point committee blinded for $\mathrm{CAC}$ scores reviewed all documents and classified the end points, as previously described. ${ }^{8}$

Statistical Analysis-The five-year rate of incident CHD (or CVD) is reported for each study, both overall and within strata defined by CAC group (i.e. 0, 1-99, 100-399, 400+). Within each study, Cox proportional hazards models were fitted relating CAC (expressed as any CAC yes/no, or log of CAC +1 , or a categorized version) to the risk of incident CHD (or CVD). Models were adjusted for age and gender, and then further adjusted for traditional cardiovascular risk factors including: presence of diabetes mellitus, smoking (never/former/ current), systolic blood pressure, use of anti-hypertensive meds, total cholesterol, high density lipoprotein, low density lipoprotein, use of lipid lowering medications, and body mass index. Five year event rates, based on logistic regression, were created for both studies and compared, after controlling for cardiovascular risk factors. We created a model to estimate 5-year event rates for a hypothetical low risk population from each cohort. We used the following risk factor profile: $50 \%$ female, age 60 , non-diabetic, never smoker, no use of lipid lowering or anti-hypertensive medications, systolic blood pressure of 120 , cholesterol $200 \mathrm{mg} / \mathrm{dl}$, HDL-C $50 \mathrm{mg} / \mathrm{dl}$, body mass index of 25 . All analyses were conducted using either SAS version 9.2 (HNR) or STATA version 11 (MESA).

\section{Results}

The final study sample consisted of 2209 participants from MESA and 3119 from HNR. Baseline demographics and descriptive statistics are presented in Table 1. Both studies included a similar proportion of men (Table 1). Mean age was slightly higher in MESA $(59.8 \pm 8.5$ yrs. in MESA vs. $58.8 \pm 7.6$ yrs. in HNR), although most of cardiovascular risk factors were worse on average in HNR, except for BMI and HDL cholesterol (Table 1). Regarding medication, intake of cholesterol medication was more than twofold higher in MESA (17.5\% in MESA vs. $8.2 \%$ in HNR), while use of anti-hypertensive medication was similar (30.4\% in MESA, 27.5\% in HNR) (Table 1). The 10 year Framingham risk score (FRS) was slightly lower in the MESA cohort (10\%) than in the HNR cohort (11\%).

\section{Cumulative events in MESA}

During 5-year follow-up, 44 (2\%) hard CHD end points occurred (including MI, resuscitated cardiac arrest, and CHD death), and 87 (3.9\%) of participants experienced CHD including revascularization among 2209 asymptomatic Caucasians. Hard CVD, defined as Hard CHD plus Stroke (not transient ischemic attack) or Stroke Death, occurred in 62 (2.8\%) (Table 2a).

\section{Cumulative events in HNR}

Over the same 5 year follow-up, 60 (1.9\%) of 3119 participants experienced hard CHD, while 101 (3.2\%) experienced CHD plus revascularization. Evaluating CVD, a total of 100 (3.2\%) of participants experienced an event. The number of coronary events and the percent event rates in each category of coronary calcification for the cohort are listed in Table $2 \mathrm{~b}$.

For both studies, event rates increased with increasing cut-points of CAC for all of the study endpoints. In MESA, the unadjusted hazard ratio (HR) for CHD including revascularization was 12.3 95\% Confidence Interval (CI) $(6.3,24.0)$ for those with CAC $>400$ compared to those with $\mathrm{CAC}=0$, and remained significant fully adjusted for age, gender and risk factors 
(HR 5.36, 95\% CI 2.46, 11.7, p<0.001), Table 3a. Similarly, In HNR, unadjusted hazard ratios for Hard CHD with revascularization were $12.6895 \% \mathrm{CI}(6.64,24.22)$, and remained significant fully adjusted for age, gender and risk factors (HR 4.88, 95\% CI 2.32, 10.24, $\mathrm{p}<0.001)$, table 4 .

CAC $>400$ remained a significant predictor in both cohorts for hard CVD, which included fatal and non-fatal stroke, as well as for hard CHD (Tables 5 and 6). Similar analysis using a cutpoint of CAC >100 was also a significant predictor in both cohorts, with similar event rates.

There were more strokes, and the absolute HR for CAC was higher in HNR when strokes and revascularization were included (although differences were not significantly different). For MESA participants with CAC >400, the HR was $9.3595 \%$ CI $(5.28,16.6)$ unadjusted, and $3.6895 \% \mathrm{CI}(1.88,7.21), \mathrm{p}<0.001$, after full adjustment, table 5. For HNR, participants with CAC >400 had a HR for CVD events of $13.8695 \%$ CI $(7.81,24.63)$ unadjusted, and $5.5895 \%$ CI $(2.92,10.65)$, adjusted, $\mathrm{p}<0.001$, Table 6.

\section{Five year Event Rates in a Healthy population}

Using logistic regression, the models created demonstrating the event rates for a healthy population is demonstrated in table 7 for both studies. All events increase in frequency with increasing cutpoints of CAC in both studies. Revascularization rates between the two studies are similar for each cutpoint of CAC, and generally, the risks of $\mathrm{CV}$ events were higher in HNR for CAC $>400$.

\section{Discussion}

These two large parallel running studies show similar HR for the association between baseline CAC and incident coronary events, but, surprisingly, more strokes in HNR. Both cohorts demonstrated very low CHD rates with zero calcium and increasing hazard ratios in both groups with CAC 100-399 and CAC >400 after multivariable adjustment for risk factors. There were some notable differences between the cohorts, as in HNR, there was a lack of significant association of incident hard CHD for categories of CAC if CAC $<400$. Both studies show that $\mathrm{CAC}>400$ is a strong predictor of events. These cohorts also demonstrated low rates of downstream revascularizations in participants who are aware (MESA) or not (HNR) of their CAC scores.

It is known that the Framingham risk prediction algorithm appears to perform fairly well in predicting CAD events in predominantly white US-American cohorts. ${ }^{14,15,16}$ However, it is also known that this model has not performed as well in European populations, reporting a substantial overestimation of CAD risk. ${ }^{17,18,19}$ Direct visualization of anatomic, preclinical atherosclerotic disease has the potential for individualized risk discrimination.

Noninvasive measurement of CAC offers a unique opportunity to compare outcomes based upon CAC in diverse populations. The predictive power of CAC for CHD and CVD was observed to be similar among two geographically separate populations in this study. The comparison of the two studies also allows a very limited assessment of the bias of informing participants of their test results, despite the potential for confounding by study (as each study is set within a different medical system). In MESA, CAC scores were revealed to participants and their physicians. This could have affected results in the MESA study in 2 ways. Because subclinical disease is asymptomatic and previously unknown to participants, it is unlikely to have had any direct impact on health behaviors at study baseline, such as lifestyle modification or medication use, that may alter relations of risk with disease. Informing patients and/or their physicians are of the results may alter this relationship, 
leading to an increase in therapies and/or behavioral changes. Thus, participants with a high CAC score who were informed (MESA only) may have had more intensive risk factor modification leading to a reduction in the number of events. The stroke data is consistent with this hypothesis but numbers are too small for it to be conclusive.

The primary difference in outcomes relates to the endpoint of CVD plus revascularizations. CAC trended towards being more predictive of events in the HNR cohort. This can be interpreted in two ways. First, it is possible that this is related to underlying risk factors, particularly blood pressure, which was $10 \mathrm{mmHg}$ higher in HNR than in MESA. Similarly, total cholesterol was $35 \mathrm{mg} / \mathrm{dl}$ higher in HNR than MESA. Hypertension has a stronger causative role in strokes than in CHD and this is one possible explanation for the differences in CAC prediction for CVD event between these two cohorts. However, the difference in prediction was maintained after controlling for risk factors and treatments, so it is more likely that higher CAC scores led to more aggressive risk factor modification (lifestyle and/ or medication), resulting in reduced power of CAC to predict events in MESA as compared to HNR. Previous studies have documented that CAC in MESA was associated with increased initiation and continuation of cardiovascular preventive medications. ${ }^{20}$

Alternatively, knowledge of a high CAC score may have biased the diagnosis of angina and, thus, could have increased downstream revascularizations. Depsite the lack of any published prospective data supporting this hypothesis, there remains a common misperception that knowledge of CAC could lead to more (potentially unnecessary) revascularizations. ${ }^{21}$ This study affords some evaluation of this potential issue by comparing the revascularization rates between populations that were aware (MESA) and not aware (HNR) of their CAC status. The 5-year revascularization rates, in these two cohorts, were relatively low. This data is consisten with randomized CAC studies such as EISNER (Early Identification of Subclinical Atherosclerosis by Noninvasive Imaging Research), ${ }^{22}$ demonstrating that within CAC scan and no-scan groups, the 2 randomized groups did not differ in 4-year utilization of stress tests, noninvasive and invasive coronary angiogram studies, and revascularization procedures.

\section{Limitations of the study}

The present study has several limitations. The main limitations of both cohorts have been discussed elsewhere. ${ }^{12}$ In the present study, we only included Caucasian participants without known coronary heart disease, so that results cannot be generalized for subjects with prevalent coronary heart disease. The MESA study previously demonstrated similar cardiovascular risk for increasing CAC scores among different race and ethnic groups. ${ }^{23} \mathrm{We}$ excluded Hispanics, African Americans and Asians in these analyses to compare to the allCaucasian HNR study. As mentioned previously, there is always a risk of confounding by medical system when comparing isolated revascularization rates between cohorts (which would not be an issue for other endpoints that are not dependent on policy decisions).

\section{Conclusions}

A comparison of two studies demonstrates that CAC performed equally well at predicting future CHD, CVD and revascularization among two independent cohorts across the Atlantic Ocean. This good prediction occurred despite some differences between the overall cohorts in treating diabetes, current smoking rates, baseline hypertension and total cholesterol. After adjustment for cardiovascular risk factors and demographic differences, the cardiovascular rates were similar between the two cohorts for different CAC cut-points. Thus, CAC is highly portable with remarkably similar outcomes across different studies, spanning different continents. 


\section{Acknowledgments}

We thank the other investigators, the staff, and the participants of the MESA study for their valuable contributions. A full list of participating MESA investigators and institutions can be found at http://www.mesa-nhlbi.org. We also thank the Heinz Nixdorf Foundation (Chairman: Dr. jur. G. Schmidt) for their generous support of this study. A full list of participating HNR investigators and institutions can be found at www.recall-studie.uni-essen.de

\section{Funding}

The MESA study is funded by the National Heart, Lung and Blood Institute of the National Institutes of Health and supported by contracts N01-HC-95159 through N01-HC-95169 from the Nationals Heart, Lung, and Blood Institute. The HNR study is funded by a contract with the private Heinz Nixdorf Foundation, Essen, Germany and undergoes continuous monitoring by governmental agencies (DLR) lead by the Bundesministerium für Bildung und Forschung (BMBF). This study is also supported by the German Ministry of Education and Science (DFG).

\section{References}

1. Chest Pain of Recent Onset: Assessment and Diagnosis of Recent Onset Chest Pain or Discomfort of Suspected Cardiac Origin. NICE Clinical Guideline. 2010; 95 at http://www.nice.org.uk/ nicemedia/live/12947/47938/47938.pdf.

2. Greenland P, Bonow RO, Brundage BH, et al. ACCF/AHA 2007 clinical expert consensus document on coronary artery calcium scoring by computed tomography in global cardiovascular risk assessment and in evaluation of patients with chest pain: a report of the American College of Cardiology Foundation Clinical Expert Consensus Task Force (ACCF/AHA Writing Committee to Update the 2000 Expert Consensus Document on Electron Beam Computed Tomography) developed in collaboration with the Society of Atherosclerosis Imaging and Prevention and the Society of Cardiovascular Computed Tomography. J Am Coll Cardiol. 2007; 49:378-402. [PubMed: 17239724]

3. Folsom AR, Kronmal RA, Detrano RC, O'Leary DH, Bild DE, Bluemke DA, Budoff MJ, Liu K, Shea S, Szklo M, Tracy RP, Watson KE, Burke GL. Coronary artery calcification compared with carotid intima-media thickness in the prediction of cardiovascular disease incidence: the MultiEthnic Study of Atherosclerosis (MESA). Arch Intern Med. 2008; 168(12):1333-1339. [PubMed: 18574091]

4. Erbel R, Möhlenkamp S, Moebus S, Schermund A, Lehmann N, Stang A, Dragano N, Grönemeyer D, Seibel R, Kälsch H, Bröcker-Preuss M, Mann K, Siegrist J, Jöckel KH. Heinz Nixdorf Recall Study Investigative Group. Coronary risk stratification, discrimination, and reclassification improvement based on quantification of subclinical coronary atherosclerosis. The Heinz Nixdorf Recall Study. J Am Coll Cardiol. 2010; 56:1397-1406. [PubMed: 20946997]

5. Bild DE, Bluemke DA, Burke GL, Detrano R, Diez Roux AV, Folsom AR, Greenland P, Jacobs DR, Kronmal R, Liu K, Nelson JC, O`Leary D, Saad MF, Shea S, Szklo M, Tracy RP. Multi-ethnic study of atherosclerosis: objectives and design. Am J Epidemiol. 2002; 156:871-881. [PubMed: 12397006]

6. Schmermund A, Möhlenkamp S, Stang A, Grönemeyer D, Seibel R, Hirche H, Mann K, Siffert W, Lauterbach K, Siegrist J, Jöckel KH, Erbel R. Assessment of clinically silent atherosclerotic disease and established and novel risk factors for predicting myocardial infarction and cardiac death in healthy middle-aged subjects: Rationale and design of the Heinz Nixdorf RECALL Study. Am Heart J. 2002; 144:212-218. [PubMed: 12177636]

7. McClelland RL, Chung H, Detrano R, Post W, Kronmal RA. from the Multi-Ethnic Study of Atherosclerosis (MESA) Distribution of Coronary Artery Calcium by Race, Gender, and Age: Results from Multi-Ethnic Study of Atherosclerosis (MESA)he. Circulation. 2006; 113:30-37. [PubMed: 16365194]

8. Erbel R, Delaney JAC, Lehmann N, et al. Signs of Subclinical Coronary Atherosclerosis in Relation to Risk Factor Distribution in the Multi-Ethnic Study of Atherosclerosis (MESA) and the Heinz Nixdorf Recall (HNR) Study. Eur Heart J. 2008; 29(22):2782-2791. [PubMed: 18845666]

9. Nasir K, Budoff MJ, Wong ND, Scheuner M, Herrington D, Arnett DK, Szklo M, Greenland P, Blumenthal RS. Family history of premature coronary heart disease and coronary artery 
calcification: Multi-Ethnic Study of Atherosclerosis (MESA). Circulation. 2007; 116:619-626. [PubMed: 17646582]

10. Executive summary of the third report of the national cholesterol education program (NCEP) expert panel on detection, evaluation, and treatment of high blood cholesterol in adults (Adult Treatment Panel III). J Am Med Assoc. 2001; 285:2486-2497.

11. Agatston AS, Janowitz WR, Hildner FJ, Zusmer NR, Viamonte M Jr. Detrano R. Quantification of coronary artery calciumusing ultrafast computed tomography. J.Am. Coll. Cardiol. 1990; 15:827832. [PubMed: 2407762]

12. Budoff MJ, McClelland RL, Chung H, Wong ND, Carr JJ, Gray MM, Blumenthal RS, Detrano RC. Reproducibility of coronary artery calcified plaque with cardiac 64-MDCT: the Multi-Ethnic Study of Atherosclerosis. AJR Am J Roentgenol. Mar; 2009 192(3):613-617. [PubMed: 19234254]

13. Thygesen K, Alpert JS, White HD, et al. Joint ESC/ACCF/AHA/WHF Task Force for the Redefinition of Myocardial Infarction. Universal definition of myocardial infarction. J Am Coll Cardiol. 2007; 50:2173-2195. [PubMed: 18036459]

14. D'Agostino RB, Grundy S, Sullivan LM, Wilson P. CHD Risk Prediction Group. Validation of the Framingham coronary heart disease prediction scores: results of a multiple ethnic groups investigation. JAMA. 2001; 286:180-187. [PubMed: 11448281]

15. Grundy SM, D'Agostino RB, Mosca L, et al. Cardiovascular risk assessment based on US cohort studies: findings from a National Heart, Lung, and Blood institute workshop. Circulation. 2001; 104:491-496. [PubMed: 11468215]

16. Kuller LH. Ethnic differences in atherosclerosis, cardiovascular disease and lipid metabolism. Curr Opin Lipidol. 2004; 15:109-113. [PubMed: 15017353]

17. Haq IU, Ramsay LE, Yeo WW, Jackson PR, Wallis EJ. Is the Framingham risk function valid for northern European populations? A comparison of methods for estimating absolute coronary risk in high risk men. Heart. 1999; 81:40-46. [PubMed: 10220543]

18. Hense HW, Schulte H, Löwel H, Assmann G, Keil U. Framingham risk function overestimates risk of coronary heart disease in men and women from Germany - results from the MONICA Augsburg and the PROCAM cohorts. Eur Heart J. 2003; 24:937-945. [PubMed: 12714025]

19. Strachan D, Rose G. Strategies of prevention revisited: effects of imprecise measurement of risk factors on the evaluation of "high-risk" and "population-based" approaches to prevention of cardiovascular disease. J Clin Epidemiol. 1991; 44(11):1187-1196. [PubMed: 1941013]

20. Nasir K, McClelland RL, Blumenthal RS, Goff DC Jr. Hoffmann U, Psaty BM, Greenland P, Kronmal RA, Budoff MJ. Coronary artery calcium in relation to initiation and continuation of cardiovascular preventive medications: The Multi-Ethnic Study of Atherosclerosis (MESA). Circ Cardiovasc Qual Outcomes. May 3.2010 (3):228-235. [PubMed: 20371760]

21. Redberg RF. What Is the Prognostic Value of a Zero Calcium Score? J Am Coll Cardiol. 2009; 55:635-636. [PubMed: 20170787]

22. Rozanski A, Gransar H, Shaw LJ, Kim J, Miranda-Peats L, Wong ND, Rana JS, Orakzai R, Hayes SW, Friedman JD, Thomson LEJ, Polk D, Min J, Budoff MJ, Berman DS. Impact of Coronary Artery Calcium Scanning on Coronary Risk Factors and Downstream Testing: The EISNER (Early Identification of Subclinical Atherosclerosis by Noninvasive Imaging Research) Prospective Randomized Trial. J Am Coll Cardiol. 2011; 57:1622-1632. [PubMed: 21439754]

23. Detrano R, Guerci AD, Carr JJ, Bild DE, Burke G, Folsom AR, Liu K, Shea S, Szklo M, Bluemke DA, O'Leary DH, Tracy R, Watson K, Wong ND, Kronmal RA. Coronary calcium as a predictor of coronary events in four racial or ethnic groups. N Engl J Med. 2008; 358:1336-1345. [PubMed: 18367736] 


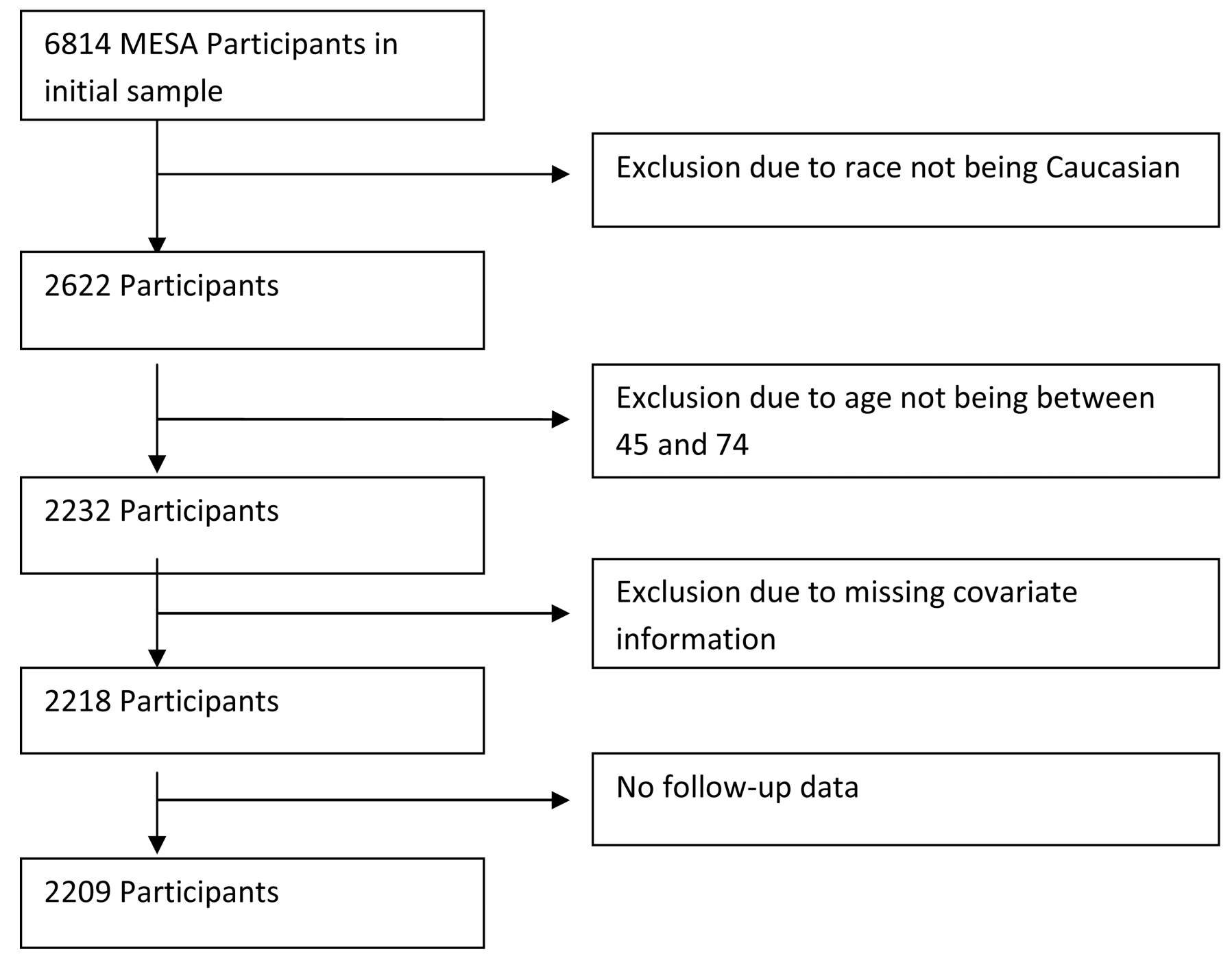

Figure 1.

Multi-Ethnic Study of Atherosclerosis (MESA) decision tree for inclusion in joint study 


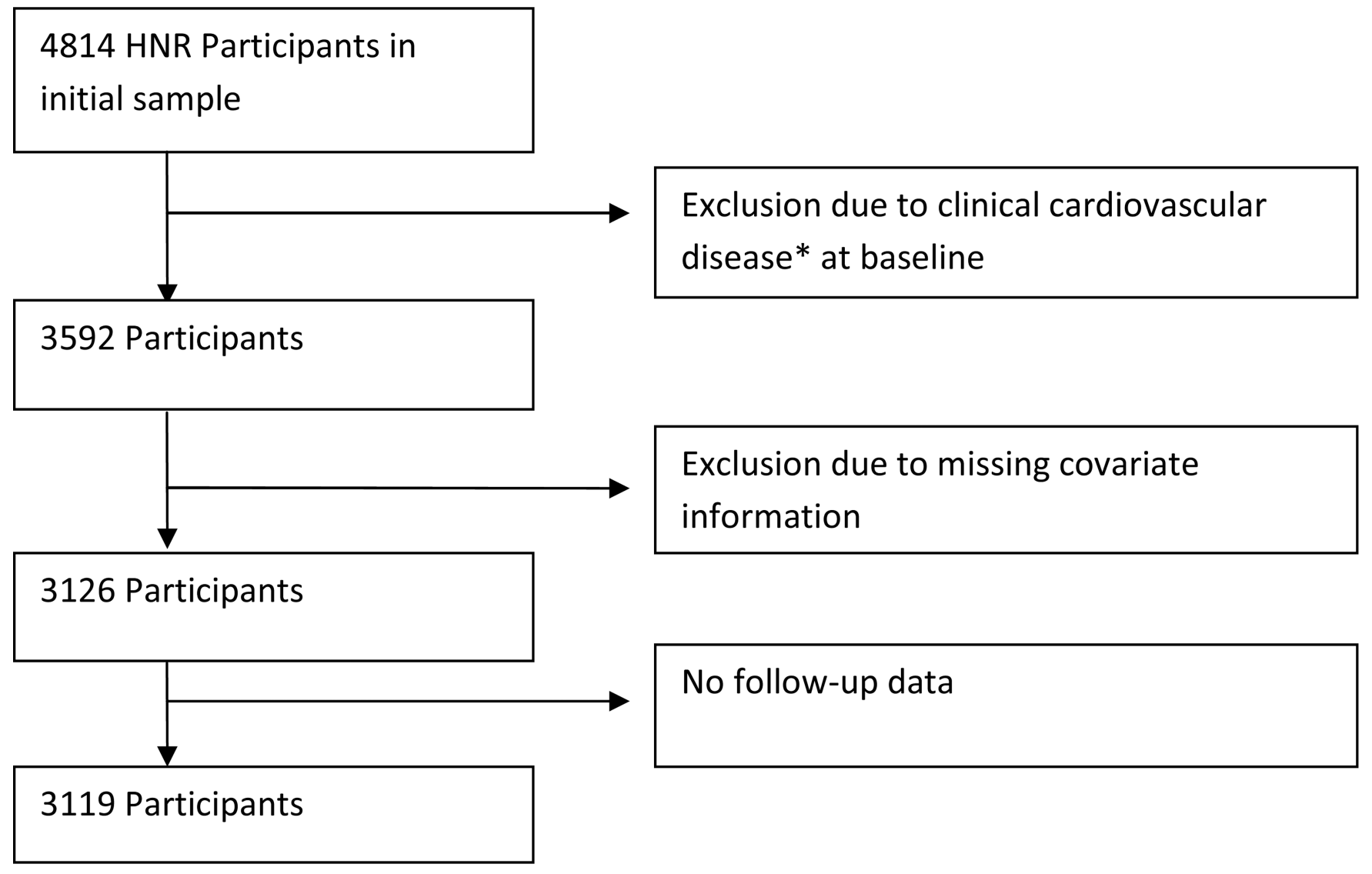

Figure 2.

Heinz Nixdorf Recall Study (HNR).decision tree for inclusion in joint study

* myocardial infarction, previous heart surgery, bypass surgery, angioplasty, valve replacement, pacemaker, atrial fibrillation, heart failure, angina or stroke 


\section{Table 1}

Descriptive Statistics for MESA and HNR

\begin{tabular}{|lcc|}
\hline & MESA (n=2209) & HNR (n=3119) \\
\hline & Mean (SD) & Mean (SD) \\
\hline Age & $59.8(8.5)$ & $58.8(7.6)$ \\
\hline Male & $47.8 \%$ & $46.8 \%$ \\
\hline Systolic Blood Pressure & $121.5(19.5)$ & $132.0(20.7)$ \\
\hline Diastolic Blood Pressure & $70.5(10.0)$ & $81.3(10.8)$ \\
\hline Body Mass Index & $27.9(5.2)$ & $27.5(4.4)$ \\
\hline Diabetes & $5.5 \%$ & $6.1 \%$ \\
\hline Total Cholesterol & $196.3(35.2)$ & $231.2(38.4)$ \\
\hline HDL Cholesterol & $52.2(15.8)$ & $59.4(17.0)$ \\
\hline Current Smoker & $12.8 \%$ & $23.5 \%$ \\
\hline Past Smoker & $44.4 \%$ & $33.2 \%$ \\
\hline Cholesterol Medication & $17.6 \%$ & $8.2 \%$ \\
\hline Blood Pressure Medication & $30.4 \%$ & $27.5 \%$ \\
\hline
\end{tabular}




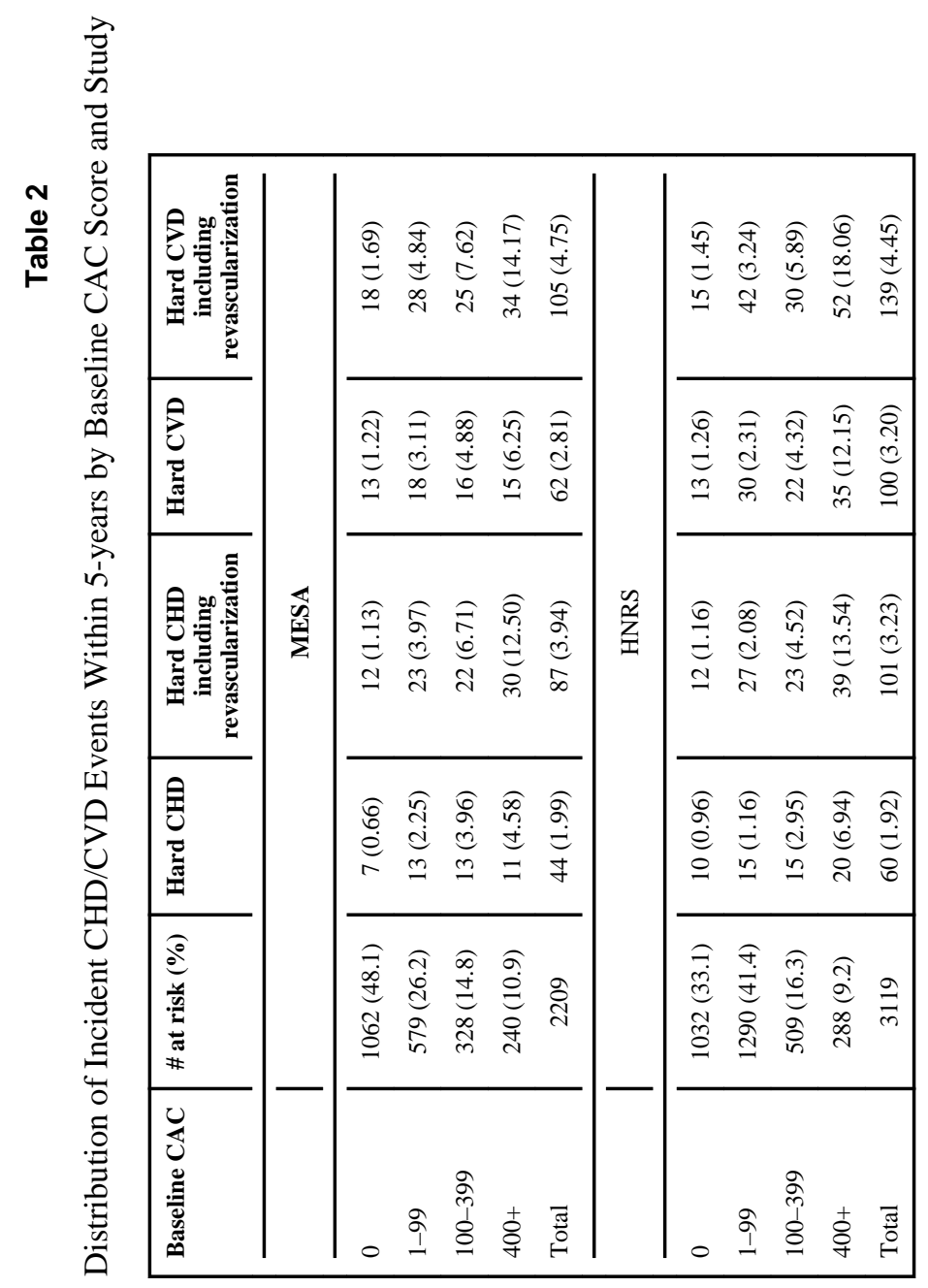




\section{Table 7}

Estimated 5-year rates for a hypothetical population with the following risk factor profile: 50\% female, age 60, non-diabetic, never smoker, no use of lipid lowering or anti-hypertensive medications, systolic blood pressure of 120 , cholesterol $200 \mathrm{mg} / \mathrm{dl}$, hdl $50 \mathrm{mg} / \mathrm{dl}$, body mass index of 25 . Estimates based on logistic regression.

\begin{tabular}{|l|c|c|c|}
\hline Event & CAC Group & $\begin{array}{c}\text { MESA } \\
\text { \% (95\% CI) }\end{array}$ & $\begin{array}{c}\text { HNR } \\
\text { (95\% CI) }\end{array}$ \\
\hline Hard CHD & 0 & $0.47(0.19-1.16)$ & $0.71(0.32-1.58)$ \\
& $1-99$ & $1.11(0.48-2.53)$ & $0.57(0.26-1.24)$ \\
& $100-399$ & $1.51(0.59-3.77)$ & $1.08(0.46-2.52)$ \\
& $400+$ & $1.53(0.56-4.14)$ & $2.09(0.86-5.00)$ \\
\hline Hard CHD incl. & 0 & $0.76(0.38-1.50)$ & $0.88(0.44-1.75)$ \\
Revascularization & $1-99$ & $1.85(1.00-3.39)$ & $1.05(0.57-1.91)$ \\
& $100-399$ & $2.40(1.20-4.76)$ & $1.69(0.86-3.30)$ \\
Hard CVD & $400+$ & $4.05(2.02-7.96)$ & $4.32(2.19-8.33)$ \\
\hline Isolated Revascularization * & 0 & $0.88(0.44-1.75)$ & $0.94(0.47-1.85)$ \\
& $1-99$ & $1.52(0.76-3.01)$ & $1.15(0.63-2.09)$ \\
& $100-399$ & $1.67(0.74-3.71)$ & $1.59(0.80-3.14)$ \\
Hard CVD incl. & $400+$ & $1.85(0.78-4.31)$ & $3.97(2.00-7.70)$ \\
\hline Revascularization & $0.72(0.29-1.77)$ & $0.67(0.31-1.43)$ \\
& $1-99$ & $1.12(0.62-1.99)$ & $1.09(0.59-2.01)$ \\
& $100-399$ & $2.15(1.23-3.74)$ & $1.62(0.97-2.70)$ \\
& $400+$ & $4.20(2.21-7.86)$ & $6.12(3.45-10.63)$ \\
& $0.79(0.28-2.24)$ & $0.89(0.37-2.13)$ \\
& $100+$ & $2.27(0.85-5.89)$ & $2.63(1.10-6.14)$ \\
\hline
\end{tabular}

isolated revascularization means that it was not preceded by a hard CHD event 